\title{
Long-Term Use of Aldosterone-Receptor Antagonists in Uncontrolled Hypertension: A Retrospective Analysis
}

\author{
Pieter M. Jansen, ${ }^{1}$ Koen Verdonk, ${ }^{1}$ Ben P. Imholz, ${ }^{2}$ A. H. Jan Danser, ${ }^{1}$ \\ and Anton H. van den Meiracker ${ }^{1}$ \\ ${ }^{1}$ Division of Pharmacology, Vascular and Metabolic Diseases, Department of Internal Medicine, Erasmus Medical Center,
3015 CE Rotterdam, The Netherlands
${ }^{2}$ Department of Internal Medicine, TweeSteden Hospital, 5141 BM Waalwijk, The Netherlands
}

Correspondence should be addressed to Anton H. van den Meiracker, a.vandenmeiracker@erasmusmc.nl

Received 30 September 2010; Accepted 24 January 2011

Academic Editor: Alan Gradman

Copyright ( $) 2011$ Pieter M. Jansen et al. This is an open access article distributed under the Creative Commons Attribution License, which permits unrestricted use, distribution, and reproduction in any medium, provided the original work is properly cited.

\begin{abstract}
Background. The long-term efficacy of aldosterone-receptor antagonists (ARAs) as add-on treatment in uncontrolled hypertension has not yet been reported. Methods. Data from 123 patients (21 with primary aldosteronism, 102 with essential hypertension) with difficult-to-treat hypertension who received an ARA between May 2005 and September 2009 were analyzed retrospectively for their blood pressure (BP) and biochemical response at first followup after start with ARA and the last follow-up available. Results. Systolic BP decreased by $22 \pm 20$ and diastolic BP by $9.4 \pm 12 \mathrm{mmHg}$ after a median treatment duration of 25 months. In patients that received treatment $>5$ years, $\mathrm{SBP}$ was $33 \pm 20$ and $\mathrm{DBP}$ was $16 \pm 13 \mathrm{mmHg}$ lower than at baseline. Multivariate analysis revealed that baseline BP and follow-up duration were positively correlated with BP response. Conclusion. Add-on ARA treatment in difficult-to-treat hypertension results in a profound and sustained BP reduction.
\end{abstract}

\section{Introduction}

Aldosterone-receptor antagonists (ARAs) have been shown to be effective in blood pressure (BP) reduction [111], but until recently their use was mainly limited to certain conditions such as liver cirrhosis, heart failure, and primary aldosteronism (PA). With the recognition of $\mathrm{PA}$ as a common cause of resistant hypertension [12], a renewed interest in the use of ARAs in hypertension has emerged. However, aldosterone has also shown to be an important factor in other forms of resistant hypertension. In patients with elevated aldosterone-to-renin ratios (ARRs) and plasma aldosterone levels, but without genuine PA based on suppression testing, BP control was harder to achieve than in essential hypertensives (EHs) [13]. Furthermore, a proportion of patients treated with angiotensinconverting enzyme inhibitors (ACE-Is) or angiotensin receptor blockers (ARBs) show aldosterone breakthrough [14-16], contributing to therapy resistance by partly counteracting the intended blockade of the renin-angiotensin-aldosteronsystem (RAAS).
The use of ARAs in resistant hypertension, therefore, seems rational, and several publications have pointed out the potential of aldosterone blockade in difficult-to-treat or resistant hypertension [17-24]. In many of these studies, the addition of spironolactone resulted in an impressive drop in systolic BP (SBP) of up to $25 \mathrm{mmHg}$ and $12 \mathrm{mmHg}$ in diastolic BP (DBP). However, most of these studies were either open label $[17,18,20,22]$, or retrospective $[19,21$, $23]$ in design. One randomized, placebo-controlled, doubleblind trial was performed comparing spironolactone with amiloride, the combination of both drugs, and placebo in black hypertensive patients with uncontrolled hypertension despite treatment with at least a diuretic and a calciumchannel-blocker [24]. Interestingly, the $\mathrm{BP}$ response was considerably smaller than in the aforementioned studies $(-7.3$ in SBP and $-3.3 \mathrm{mmHg}$ in DBP for spironolactone versus placebo). De Souza et al. recently performed an open-label, prospective study on the BP-lowering benefits of spironolactone in patients with resistant hypertension. By using 24-hour ambulatory BP measurements, at least part of the potential white coat and placebo effect could be 
accounted for. Twenty-four-hour SBP and DBP decreased by 16 and $9 \mathrm{mmHg}$, respectively, after a median treatment duration of 7 months, and in a subgroup, the persistence of this effect was confirmed up to 15 months [22]. So far, longer followup periods have not been reported and although a persistence of the effect in the long run is expected, this remains to be confirmed.

Predicting factors for the BP response to ARA treatment have been identified in several studies. Lower serum potassium levels were pointed out by several groups to be associated with a larger decrease in BP $[19,21,22,25]$. Most studies found no relation between plasma renin concentration or activity and the BP lowering response to ARAs $[5,9,17,26]$. Also neither plasma aldosterone levels nor ARR levels seem to predict the BP-lowering effect $[22,26]$, although this could have been caused by the interfering effects of multidrug antihypertensive regimens on the ARR in these patients [25]. Other factors possibly associated with a better response are the absence of diabetes [23], higher waist circumference, lower aortic pulse wave velocity [22], and a lower baseline high-density lipoprotein (HDL) cholesterol [21].

ARAs have been prescribed in our clinic to patients with difficult-to-treat hypertension for a long time now, often with good results even after many years. This study aims to retrospectively characterize the long-term response to ARA treatment in patients with difficult-to-treat hypertension and to identify factors associated with this response.

\section{Methods}

2.1. Patients. All patients who visited the outpatient hypertension clinic of the Erasmus Medical Center in Rotterdam and the TweeSteden Hospital in Waalwijk, the Netherlands, between May 2005 and September 2009 were screened for their eligibility for the study. Patients were selected when they had uncontrolled hypertension (BP > 140/90 $\mathrm{mmHg}$, or $>130 / 80 \mathrm{mmHg}$ for patients with diabetes mellitus (DM) or manifest cardiovascular disease) despite the use of at least two antihypertensive drugs and were put on spironolactone or eplerenone during the study period. Patients who were already using an ARA when referred to our clinic were excluded. Patients of whom insufficient data was available to meet the primary objective (for instance insufficient data on medication use or the absence of a BP measurement at the start of treatment or last followup) or patients who were prescribed an ARA for another indication than hypertension were also excluded from the analysis.

2.2. Clinical Data. At baseline, patients' sex, height, weight, the time of diagnosis of hypertension, their antihypertensive medication, their family history, and the presence or absence of diabetes at the start of ARA treatment were collected from patient files. Their electrocardiograms (ECGs), when not taken longer than one year before start of treatment, were scored for the presence of left ventricular hypertrophy (LVH) according to the Sokolow-Lyon criteria. The presence or absence of PA was based on the clinical judgement by their physician.
At baseline, at first followup (i.e., the first followup visit that BP was measured after start of ARA treatment), and at the end of followup (i.e., the date that ARA treatment was permanently discontinued or the last visit before the end of data collection), the following parameters were recorded: BP, serum sodium, potassium, urea, creatinine, uric acid, glucose, total cholesterol, high-density lipoprotein (HDL), low-density lipoprotein (LDL), and hemoglobin and hematocrit, and plasma renin and aldosterone levels, where available.

BP measurements were taken in triplicate at an interval of five minutes with a semiautomatic BP measuring device after a rest of five minutes in sitting position. The mean of these measurements was used in the analysis.

Biochemical measurements were taken on the visit day or the nearest previous moment.

Plasma renin concentrations (PRCs) were assessed using an immunoradiometric assay (Renin III, Cisbio, Gif-surYvette, France). Plasma aldosterone concentrations (PACs) were measured with a radioimmunoassay (Coat-a Count, Diagnostics Product Corporation, LA, CA, USA). Hyperkalemia was defined as serum potassium levels exceeding $5.5 \mathrm{mmol} / \mathrm{L}$.

2.3. Data Analysis. Statistical analyses were performed in SPSS 17.0 for Windows.

Main effects at first followup and end of followup were calculated. Furthermore, to assess the long-term efficacy of treatment, patients were stratified based on the duration of followup into the following categories: $<1$ year, 1-5 years, and $>5$ years followup.

Values are expressed as mean $\pm \mathrm{SD}$, or as median and range when not normally distributed. Medication use was quantified by adding up the total number of different drugs, as well as by assessing the defined daily doses (DDDs) per drug and for total drug use according to the World Health Organization Anatomical Therapeutic Chemical (ATC) index [27]. Differences within subjects were tested using paired Student's $t$-tests for two groups and one-way analysis of variance (ANOVA) for repeated measurements for more groups. Between-subjects differences were tested with unpaired $t$-tests for two groups and one-way ANOVA for more groups. For values that were abnormally distributed, nonparametric tests were used (Mann-Whitney $U$ test and Wilcoxon Signed Ranks test). Differences in proportions were tested with a chi-square test.

Patients with PA were excluded for regression analysis. A univariate linear regression analysis was performed to identify potential determinants of the BP response. Significant parameters were subsequently tested in a multivariate linear regression analysis. This model was further adjusted for age and sex.

\section{Results}

3.1. Study Population. A total of 175 patients were prescribed an ARA during the study period. Fifty-two patients were excluded: 39 because of insufficient data, 5 because our 
TABLE 1: Baseline characteristics of the study population.

\begin{tabular}{|c|c|c|c|c|}
\hline & Total & $\mathrm{EH}$ & PA & $P$-value \\
\hline Number & 123 & 102 & 21 & \\
\hline Age (years) & $56.6 \pm 10.7$ & $56.7 \pm 11.2$ & $56.5 \pm 8.2$ & .959 \\
\hline Male (\%) & 60.1 & 56.9 & 76.2 & .099 \\
\hline BMI $\left(\mathrm{kg} / \mathrm{m}^{2}\right)$ & $29.4 \pm 5.0$ & $29.3 \pm 5.0$ & $30.1 \pm 5.2$ & .537 \\
\hline SBP (mmHg) & $159.7 \pm 19.1$ & $158.4 \pm 18.3$ & $166.0 \pm 21.7$ & .094 \\
\hline DBP (mmHg) & $93.3 \pm 12.2$ & $92.7 \pm 12.5$ & $96.0 \pm 10.8$ & .268 \\
\hline Time since diagnosis (years) & $10.0(0-50)$ & $10.0(0-50)$ & $7.5(1.0-34)$ & .319 \\
\hline Age at diagnosis (years) & $42.0 \pm 13.0$ & $41.5 \pm 13.3$ & $44.7 \pm 11.4$ & .335 \\
\hline Nr. of antihypertensives & $3(2-6)$ & $3(2-6)$ & $3(2-5)$ & .071 \\
\hline DDD & $5.0(1.25-13.0)$ & $5.0(1.25-13.0)$ & $3.7(1.5-10.0)$ & .117 \\
\hline $\mathrm{DM}(\%)$ & 22.8 & 23.2 & 21.1 & .842 \\
\hline LVH $(\%)$ & 28.5 & 26.5 & 38.1 & .125 \\
\hline Family history of HT & 52.0 & 53.9 & 42.9 & .355 \\
\hline Serum sodium $(\mathrm{mmol} / \mathrm{L})$ & $141.5 \pm 2.7$ & $141.2 \pm 2.8$ & $143.0 \pm 2.14$ & .008 \\
\hline Serum potassium $(\mathrm{mmol} / \mathrm{L})$ & $3.9 \pm 0.6$ & $4.0 \pm 0.6$ & $3.4 \pm 0.5$ & $<.001$ \\
\hline Serum creatinine $(\mu \mathrm{mol} / \mathrm{L})$ & $83.8 \pm 20.1$ & $83.8 \pm 21.1$ & $84.1 \pm 14.4$ & .959 \\
\hline Serum uric acid (mmol/L) & $0.36 \pm 0.08$ & $0.37 \pm 0.08$ & $0.34 \pm 0.08$ & .134 \\
\hline Hemoglobin $(\mathrm{mmol} / \mathrm{L})$ & $8.9 \pm 0.82$ & $8.8 \pm 0.8$ & $9.5 \pm 0.6$ & .001 \\
\hline Hematocrit $(\%)$ & $0.42 \pm 0.04$ & $41.3 \pm 3.6$ & $45.3 \pm 2.1$ & .003 \\
\hline Cholesterol (mmol/L) & $5.31 \pm 0.96$ & $5.27 \pm 0.97$ & $5.55 \pm 0.90$ & .345 \\
\hline $\mathrm{HDL}(\mathrm{mmol} / \mathrm{L})$ & $1.35 \pm 0.42$ & $1.37 \pm 0.41$ & $1.26 \pm 0.44$ & .347 \\
\hline $\mathrm{LDL}(\mathrm{mmol} / \mathrm{L})$ & $3.37 \pm 1.02$ & $3.41 \pm 1.02$ & $3.16 \pm 1.03$ & .407 \\
\hline Glucose $(\mathrm{mmol} / \mathrm{L})$ & $5.5 \pm 1.6$ & $5.5 \pm 1.6$ & $5.5 \pm 1.8$ & .943 \\
\hline $\operatorname{ACR}(\mathrm{g} / \mathrm{mol})$ & $2.19(0.95-12.4)$ & $2.19(0.15-453.8)$ & $1.96(0.37-592.0)$ & .518 \\
\hline $\mathrm{PAC}(\mathrm{pmol} / \mathrm{L})$ & $282.5(2.8-4172)$ & $224.4(2.8-4172)$ & $548.5(199-2282)$ & $P<.001$ \\
\hline $\mathrm{PRC}(\mathrm{mU} / \mathrm{L})$ & $13.9(1.0-4374)$ & $19.8(1.0-4374)$ & $5.8(1.8-18.9)$ & $P<.001$ \\
\hline $\operatorname{ARR}(\mathrm{pmol} / \mathrm{mU})$ & $19.4(0.3-1087)$ & $9.5(0.3-781)$ & $82.7(17.4-1087)$ & $P<.001$ \\
\hline
\end{tabular}

(EH: essential hypertension; PA: primary aldosteronism; BMI: body mass index; SBP: systolic blood pressure; DBP: diastolic blood pressure; DDD: defined daily dose; DM: diabetes mellitus; LVH: left ventricular hypertrophy; HT: hypertension; HDL: high-density lipoprotein; LDL: low-density lipoprotein; ACR: urinary albumin-to-creatinine ratio; PAC: plasma aldosterone concentration; PRC: plasma renin concentration; ARR: aldosterone-to-renin ratio).

criteria for difficult-to-treat hypertension were not met, 3 because of questionable treatment adherence, 2 because of a followup duration less than a month, 1 because baseline BP measurement was not performed with a semi-automatic BP measuring device, and 1 because an ARA was prescribed because of another indication than hypertension. In total, 123 patients were included in the analysis with a mean age of $56.6 \pm 10.7$ years. The median duration between diagnosis and start of ARA treatment was 10 years (range 050 years). The median number of different antihypertensive agents was 3 (total DDD 5.0). Twenty-three percent of patients had DM, and 29 percent had LVH. Twenty-one patients were diagnosed as having PA by their physician. The baseline characteristics of all patients and of the $\mathrm{EH}$ and PA subgroups are shown in Table 1. Serum potassium levels were lower in patients with PA than with $\mathrm{EH}(3.4 \mathrm{mmol} / \mathrm{L}$ versus $4.0 \mathrm{mmol} / \mathrm{L}$ in $\mathrm{EH}, P<.001)$. Serum sodium levels were higher in patients with PA than with EH (143 versus $141 \mathrm{mmol} / \mathrm{L}, P<.001)$.
As expected, PRC was lower in PA than in $\mathrm{EH}$ patients (5.8 versus $19.8 \mathrm{mU} / \mathrm{L}, P<.001$ ). PAC and ARR were higher in PA patients (548.5 versus $224.4 \mathrm{pmol} / \mathrm{L}(P<.001)$ for PAC, and 82.7 versus $9.5 \mathrm{pmol} / \mathrm{mU}(P<.001)$ for ARR).

Values of haemoglobin and hematocrit were also higher in PA than in $\mathrm{EH}$ patients.

3.2. Treatment. Ninety-four patients started on spironolactone treatment with a median dose of $50 \mathrm{mg}$ daily (range 12.5-100 mg). Twenty-nine patients started on eplerenone with a median dose of $50 \mathrm{mg}$ (range $25-50 \mathrm{mg}$ ). Total starting DDD of ARA was 0.67 (range 0.17-1.33). At the end of followup 91 patients were on spironolactone with a median dose of $25 \mathrm{mg}$ (range 12.5-100 mg) and 32 patients on eplerenone (median dose $50 \mathrm{mg}$, range $25-100 \mathrm{mg}$ ). Median ARA DDD at end of followup was 0.67 (range 0.17-2.00). Median treatment duration at first followup was 8 weeks (range 1-66 weeks). The median treatment duration at end of followup was 25 months (range 1-144 months). 


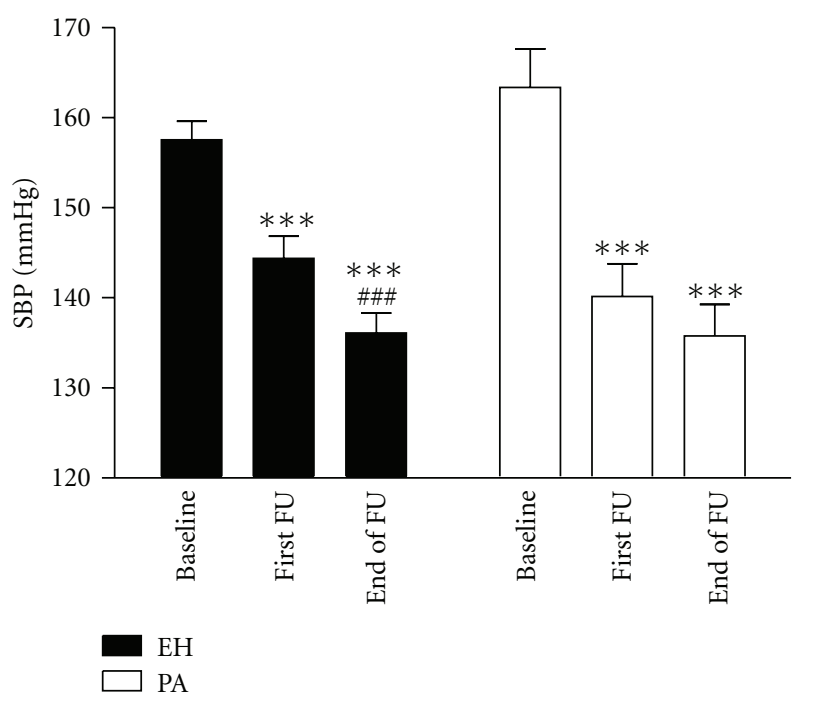

(a)

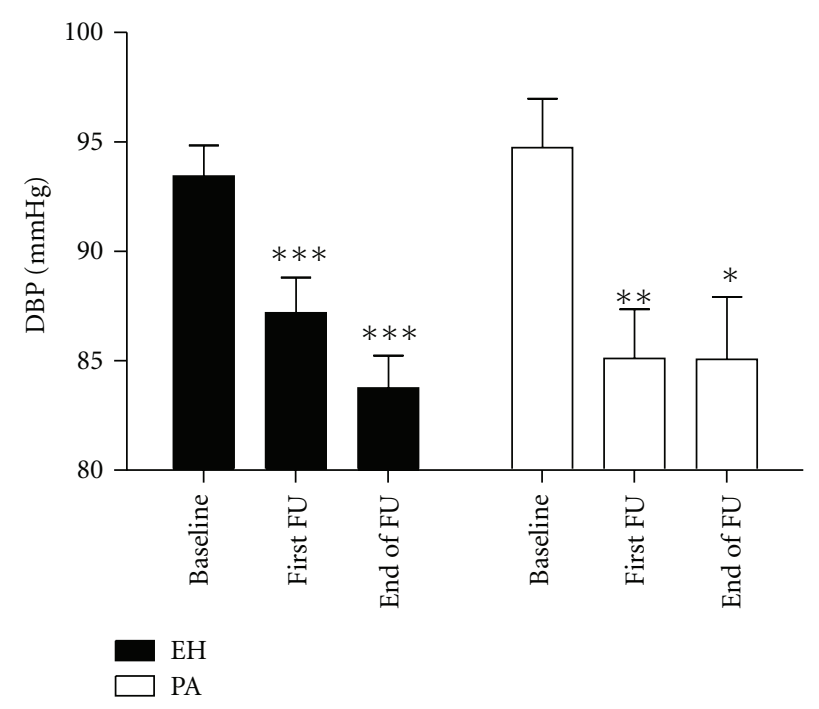

(b)

FIGURE 1: Systolic (SBP) and diastolic (DBP) blood pressure before start with an aldosterone-receptor antagonist (baseline), at first followup $(\mathrm{FU})$, and at the end of $\mathrm{FU}$ in patients with essential hypertension $(\mathrm{EH})$ and primary aldosteronism (PA). Overall trend was tested with one-way ANOVA for repeated measurements $(P<.001$ for all groups, except for DBP in the PA group $(P=.001))$. Indicated significance levels are for differences between groups after adjustment for multiple comparisons ( ${ }^{*}$ compared to baseline; ${ }^{*}$ compared to first FU).

TABLE 2: Changes in biochemical parameters at first followup and end of followup after start of treatment with an aldosterone-receptor antagonist for patients with essential hypertension (EH) and primary aldosteronism (PA). (Values were tested with one-way ANOVA for repeated measurements. Indicated $P$ values are for differences between baseline and first followup (a), first and last followup (b), and baseline and last followup (c) after Bonferroni adjustment; $n$ represents the number of patients with measurements at all three time points).

\begin{tabular}{|c|c|c|c|c|c|c|c|c|}
\hline & & $n$ & Baseline & $P$ value $^{\mathrm{a}}$ & First FU & $P$ value $^{\mathrm{b}}$ & End of FU & $P$ value ${ }^{\mathrm{c}}$ \\
\hline \multirow{3}{*}{$\mathrm{EH}$} & Serum sodium $(\mathrm{mmol} / \mathrm{L})$ & 66 & $141.2 \pm 2.7$ & 1.000 & $140.9 \pm 3.0$ & .927 & $141.3 \pm 3.2$ & 1.000 \\
\hline & Serum potassium $(\mathrm{mmol} / \mathrm{L})$ & 80 & $4.0 \pm 0.6$ & $<.001$ & $4.4 \pm 0.6$ & 1.000 & $4.4 \pm 0.5$ & $<.001$ \\
\hline & Serum creatinine $(\mu \mathrm{mol} / \mathrm{L})$ & 78 & $84.6 \pm 20.8$ & $<.001$ & $90.8 \pm 24.7$ & .355 & $93.6 \pm 26.2$ & $<.001$ \\
\hline \multirow{3}{*}{ PA } & Serum sodium (mmol/L) & 17 & $142.9 \pm 2.1$ & .015 & $141.0 \pm 3.4$ & .103 & $142.7 \pm 3.4$ & 1.000 \\
\hline & Serum potassium $(\mathrm{mmol} / \mathrm{L})$ & 19 & $3.4 \pm 0.5$ & $<.001$ & $4.3 \pm 0.5$ & 1.000 & $4.3 \pm 0.5$ & $<.001$ \\
\hline & Serum creatinine $(\mu \mathrm{mol} / \mathrm{L})$ & 18 & $85.7 \pm 14.1$ & .011 & $96.1 \pm 22.6$ & 1.000 & $95.7 \pm 19.6$ & .169 \\
\hline
\end{tabular}

3.3. Main Effects of ARA Treatment. The BP levels at first followup and at the end of followup are shown in Figure 1. In $\mathrm{EH}$ patients, $\mathrm{BP}$ decreased by $13 \pm 1.8 \mathrm{mmHg}$ systolically and $6.2 \pm 1.0 \mathrm{mmHg}$ diastolically at first followup, and by $21 \pm 2.1$ and $9.7 \pm 1.4 \mathrm{mmHg}$ at the end of followup. In PA patients, SBP had decreased by $23 \pm 4.8 \mathrm{mmHg}$ and DBP by $9.6 \pm 2.5 \mathrm{mmHg}$ at first followup and by $28 \pm 4.9$ and $9.7 \pm 3.1 \mathrm{mmHg}$ at the end of followup. Changes in BP were not significantly different for EH and PA patients at both time points, although a trend existed towards a larger SBP decrease at first followup in the PA group $(P=.063)$.

Serum potassium and creatinine levels increased significantly after start of ARA treatment for both EH and PA patients. Furthermore, in PA patients, serum sodium was significantly lower at first followup compared to baseline (Table 2).

At baseline, PA and EH patients used a median number of 3 antihypertensive drugs (range 2-6). At the end of followup, the number of drugs had increased to 4 (range 1 to $7, P<.001)$. However, when expressed in DDD, the total amount of antihypertensive drugs remained unchanged (5 DDD at baseline versus 4.5 at end of followup, $P=.459$ ). Also in the EH subgroup, the number of antihypertensive drugs increased from 3 to $4(P<.001)$, with a nonsignificant decrease in DDD (5 DDD at baseline against 4.6 at end of followup, $P=.663$ ). In PA patients, there was no significant change in number of antihypertensive drugs (3 versus 3 , $P=.317$ ) or DDD (3.66 versus $3.83, P=.407$ ).

3.4. Stratification to Followup Duration. Because of the wide variation in followup duration and to better assess the long-term efficacy of ARA treatment, patients were stratified according to their treatment followup. The following categories were formed: $0-1$ year, $1-5$ years, and $>5$ years. Number of patients in these categories were 33, 49, and 


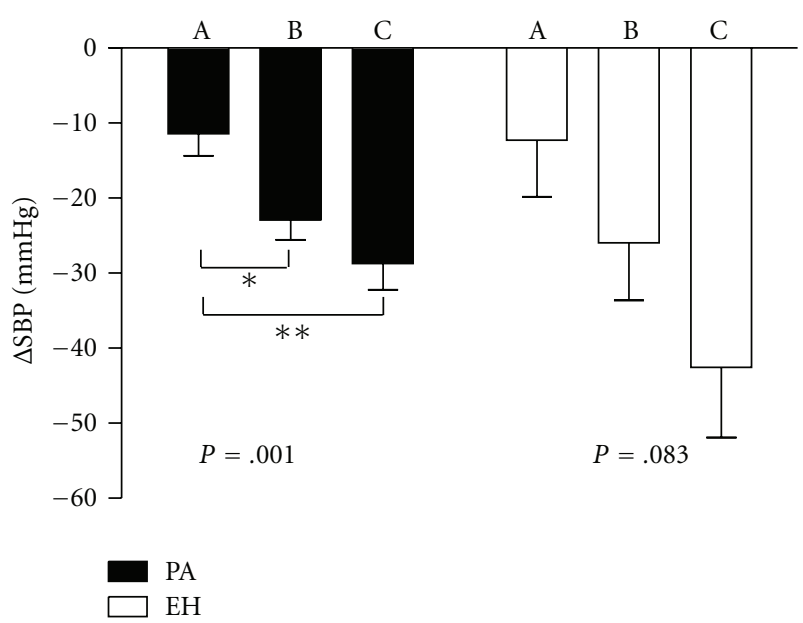

(a)

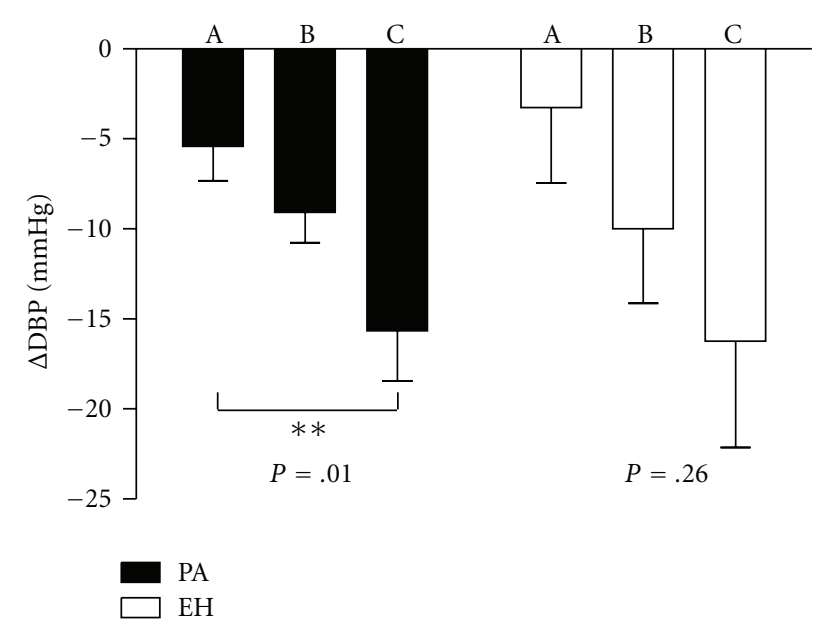

(b)

FIGURE 2: Changes in systolic (SBP) and diastolic (DBP) blood pressure at the end of followup compared to baseline for three categories of followup duration (A $<1$ year; B 1-5 years; $C>5$ years). $P$ values are for trend tested with one-way ANOVA; indicated significance levels are for differences between groups after adjustment for multiple comparisons. (EH: essential hypertension; PA: primary aldosteronism).

20, respectively, for $\mathrm{EH}$ patients, and 5, 8 , and 8 for $\mathrm{PA}$ patients. In Figure 2, blood pressure reduction is shown for the three categories of followup duration. In EH patients larger responses were seen with longer followup duration $(P=.001$ for $\triangle \mathrm{SBP}$ and $P=.01$ for $\triangle \mathrm{DBP}$ with oneway ANOVA). In PA patients, a similar trend was seen. The overall trends were not different for $\mathrm{EH}$ and PA patients $(P=.467$ for $\triangle \mathrm{SBP}$ and $P=.907$ for $\triangle \mathrm{DBP}$ at two-way ANOVA).

To investigate whether the reduction in BP was merely a result of a greater number of antihypertensive drugs than a specific effect of ARA treatment, baseline and end-offollowup BP is shown in relation to medication use for EH (Figure 3) and PA (Figure 4). The proportion of total DDD that consisted of ARA treatment is separately indicated. These figures show that at longer followup, BP further decreased, while the total DDD remained unchanged. In $\mathrm{EH}$ patients, the percentage of total DDD consisting of an ARA significantly increased from $9.1 \%$ to $14.2 \%(P<$ .001 ) in the 1-5-year followup group. In PA patients, the relative contribution of ARA to total DDD increased from $14.9 \%$ to $22.4 \%$ in the $1-5$-year followup group $(P=.050)$ and from $14.9 \%$ to $31.9 \%$ in the $>5$ years followup group $(P=.018)$.

3.5. Predictors for the Blood Pressure Response. The main clinical parameters were tested for their potential association with SBP as well as DBP response at first and last followup by univariate regression analysis (with the change in $\mathrm{BP}$ being negative). Table 3 shows the beta coefficients of all parameters that were significantly associated with $\mathrm{BP}$ change in any of the four groups, as well as those considered relevant based on earlier reports. At first followup, the sodium/potassium ratio as well as followup duration were significantly associated with $\triangle \mathrm{SBP}$. The ARR was significantly associated with $\triangle \mathrm{DBP}$, yet with a very small and probably irrelevant regression coefficient considering the range in ARR. Interestingly, haemoglobin and hematocrit levels, total cholesterol, and LDL levels were negatively associated with blood pressure change at univariate analysis for $\triangle \mathrm{SBP}$, and the latter two also for $\triangle \mathrm{DBP}$.

At last followup, the change in BP was significantly correlated with baseline BP, urinary albumin-to-creatinine ratio (ACR), LVH, followup duration, and, for DBP, the ARR.

To identify independent predictors for BP response, the variables significantly associated in the univariate analyses were included in a multivariate linear regression analysis. In addition, the model was adjusted for age and sex. The regression coefficients and significance levels are shown in Table 4. Unfortunately, hemoglobin, hematocrit, total cholesterol, LDL, LVH, and the ARR could not be included in the analysis because numbers were too small to maintain sufficient statistical power.

At first followup, only baseline SBP seemed to be an independent predictor (borderline significant) for $\triangle \mathrm{SBP}$. For $\triangle \mathrm{DBP}$, there were no independent predictors for the response. At the end of followup, higher baseline BP and longer FU duration were independently associated with the change in BP.

3.6. Adverse Events. ARA treatment was in general well tolerated. In total, 13 adverse events were reported. Five cases of gynaecomastia were reported with spironolactone use resulting in a switch to eplerenone in 1 patient. Two cases of hyperkalemia were seen, and in two patients, a clinically relevant decrease in renal function was observed. Two patients (one on eplerenone and one on spironolactone) reported general discomfort and headache, and one patient experienced gastrointestinal discomfort, although this was probably already present before start of spironolactone. In 1 patient, the nature of the adverse event was not further specified. 


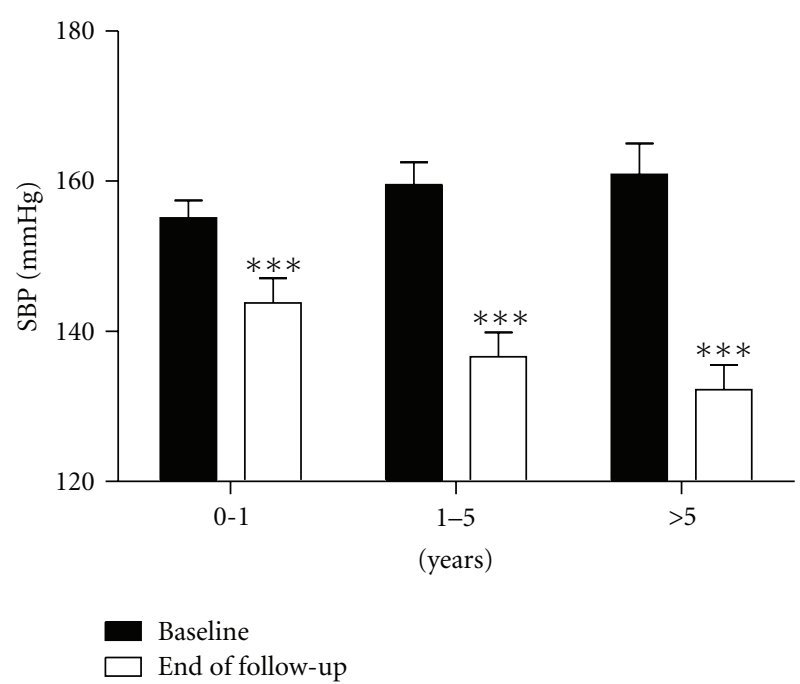

(a)

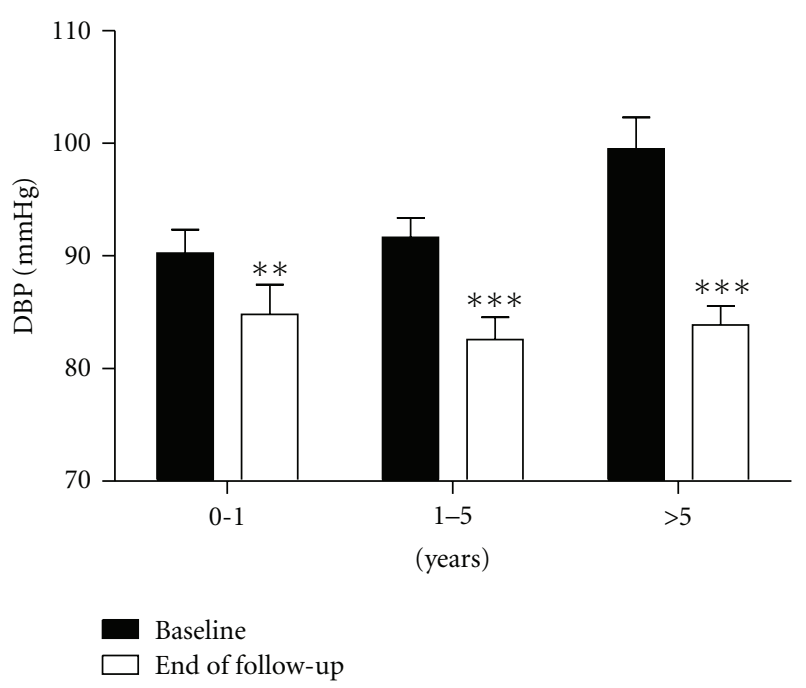

(b)

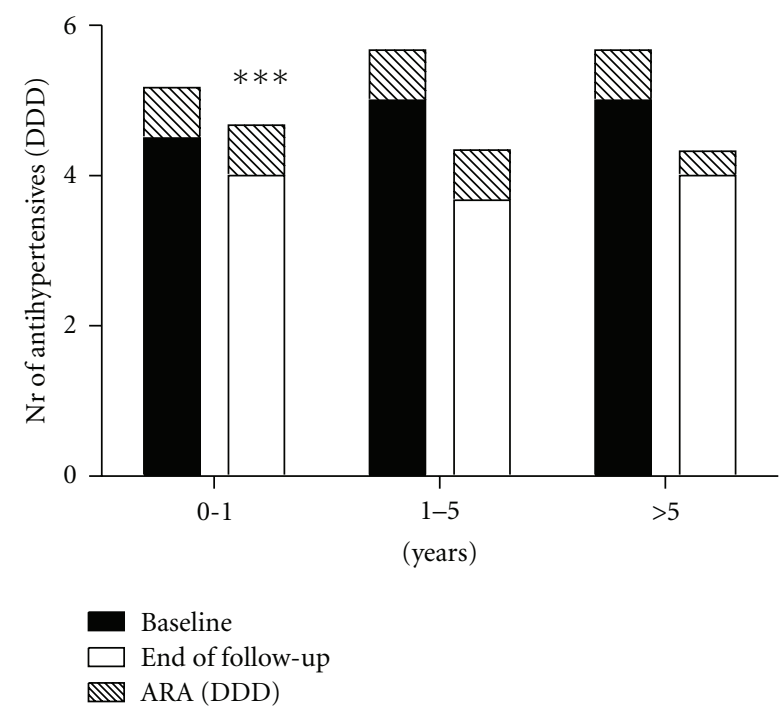

(c)

FIGURE 3: Systolic (SBP) (a), diastolic (DBP) (b) blood pressure and medication use (defined daily dose, DDD) (c) at baseline and end of followup after stratification for followup duration for patients with essential hypertension (Figure 3) and primary aldosteronism (Figure 4). Indicated in (c) is the DDD for the aldosterone-receptor antagonist (ARA). For baseline, this is added up to total DDD; at the end of followup, this is part of the total DDD since ARA was started at baseline. Differences were tested with paired $t$-test for SBP and DBP and Wilcoxon Signed Ranks test for DDD (DDD without ARA at baseline versus DDD including ARA at the end of followup).

\section{Discussion}

This study shows that the addition of aldosterone-receptor antagonists (ARAs) in patients with difficult-to-treat hypertension was highly effective in reducing SBP as well as DBP. This effect was already present at short-term followup (median followup 8 weeks) and persisted in the long run with a median followup of 25 months. The BP reduction in $\mathrm{EH}$ and PA patients was comparable, and in both groups ARA treatment resulted in a small rise in serum potassium and creatinine levels.

To assess whether the BP-lowering effect was still present after prolonged treatment, patients were stratified according to their duration of followup. We observed larger BP reductions with increasing followup, which was highly significant in EH patients. In the subgroup that had a followup of more than 5 years, SBP was $29 \mathrm{mmHg}$ and DBP $16 \mathrm{mmHg}$ lower than at baseline. In PA, a similar trend was seen although this failed to reach statistical significance, probably because of the small number of patients in each subgroup. Also in the multivariate regression analysis we observed a strong correlation between treatment duration and decrease in BP. Although it is appealing to conclude that a longer treatment duration leads to better BP control for instance by reversing target organ damage, a more likely explanation is some form of effect-bias implicating that patients with a better response 


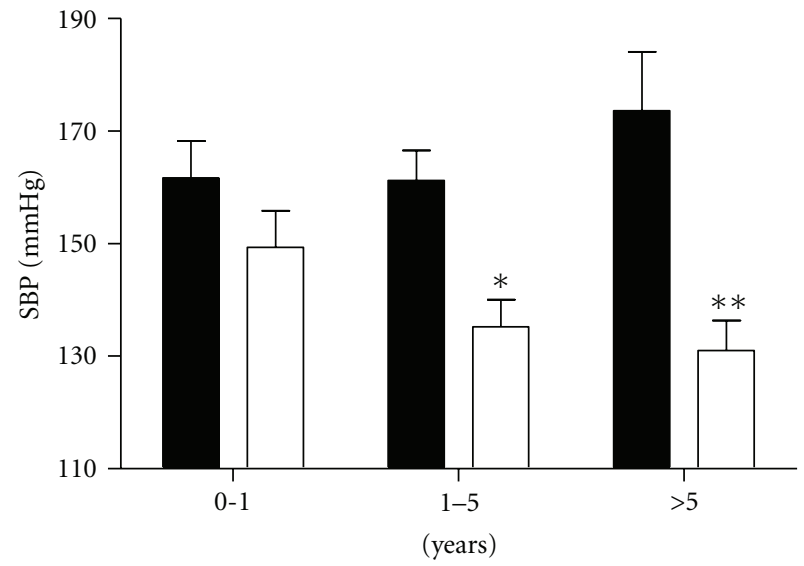

Baseline End of follow-up

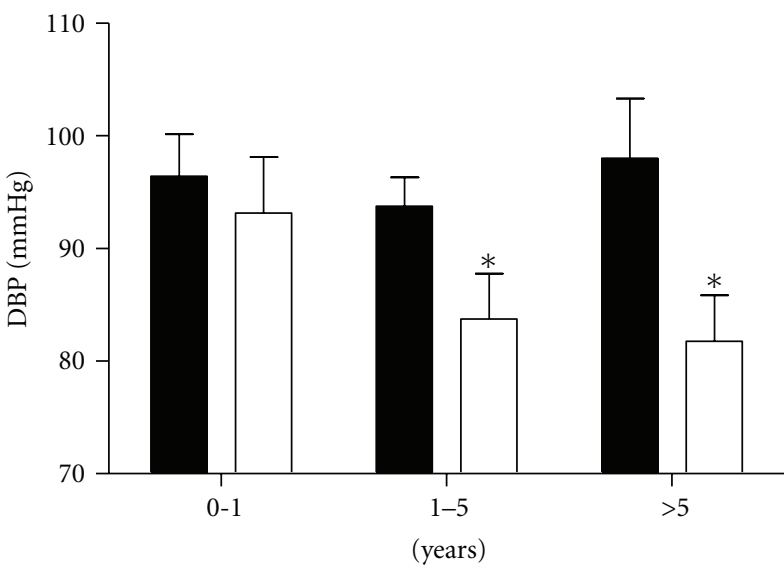

Baseline End of follow-up

(a)

(b)

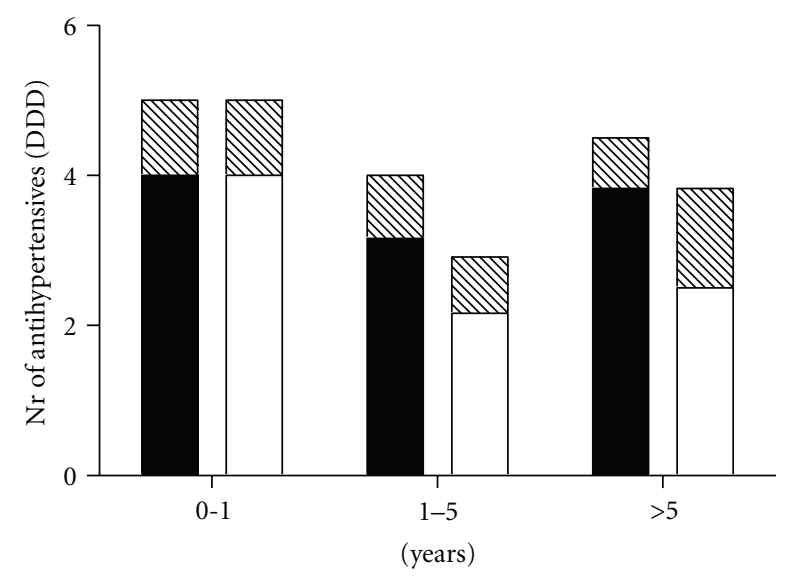

Baseline

End of follow-up

ARA (DDD)

(c)

FIGURE 4: Systolic (SBP) (a), diastolic (DBP) (b) blood pressure and medication use (defined daily dose, DDD) (c) at baseline and end of followup after stratification for followup duration for patients with essential hypertension (Figure 3) and primary aldosteronism (Figure 4). Indicated in (c) is the DDD for the aldosterone-receptor antagonist (ARA). For baseline, this is added up to total DDD; at the end of followup, this is part of the total DDD since ARA was started at baseline. Differences were tested with paired $t$-test for SBP and DBP and Wilcoxon Signed Ranks test for DDD (DDD without ARA at baseline versus DDD including ARA at the end of followup).

are more likely to receive ARA treatment for a longer period. Whether prolonged treatment leads to a better BP control requires a long-term prospective study.

Another explanation for the favourable long-term BP response could be an optimalisation of the antihypertensive medication or merely the fact that the total amount of medication increased over time. To investigate this further, $\mathrm{BP}$ values at baseline and at end of followup were shown in relation to total medication use. Although BP decreased considerably over the study period, the total amount of DDD remained virtually the same. The relative contribution of ARA treatment to total DDD increased over time. The possibility that the improved BP reduction during long- term followup is due to an increase in total amount of antihypertensive medication can therefore be excluded.

The BP responses in this study were of similar magnitude as those observed in other retrospective or open-label studies concerning add-on ARA treatment [17-21, 23]. Interestingly, in two prospective trials, BP reductions were considerably smaller than in the aforementioned studies. Saha et al. [24] studied the effect of spironolactone in black hypertensive patients with uncontrolled BP despite the use of at least a diuretic and a calcium-channel-blocker in a randomized, placebo-controlled manner and reported a reduction of $7.3 \mathrm{mmHg}$ in SBP and $3.3 \mathrm{mmHg}$ in DBP. In a recent study, De Souza et al. [22] assessed the effect of open-label 


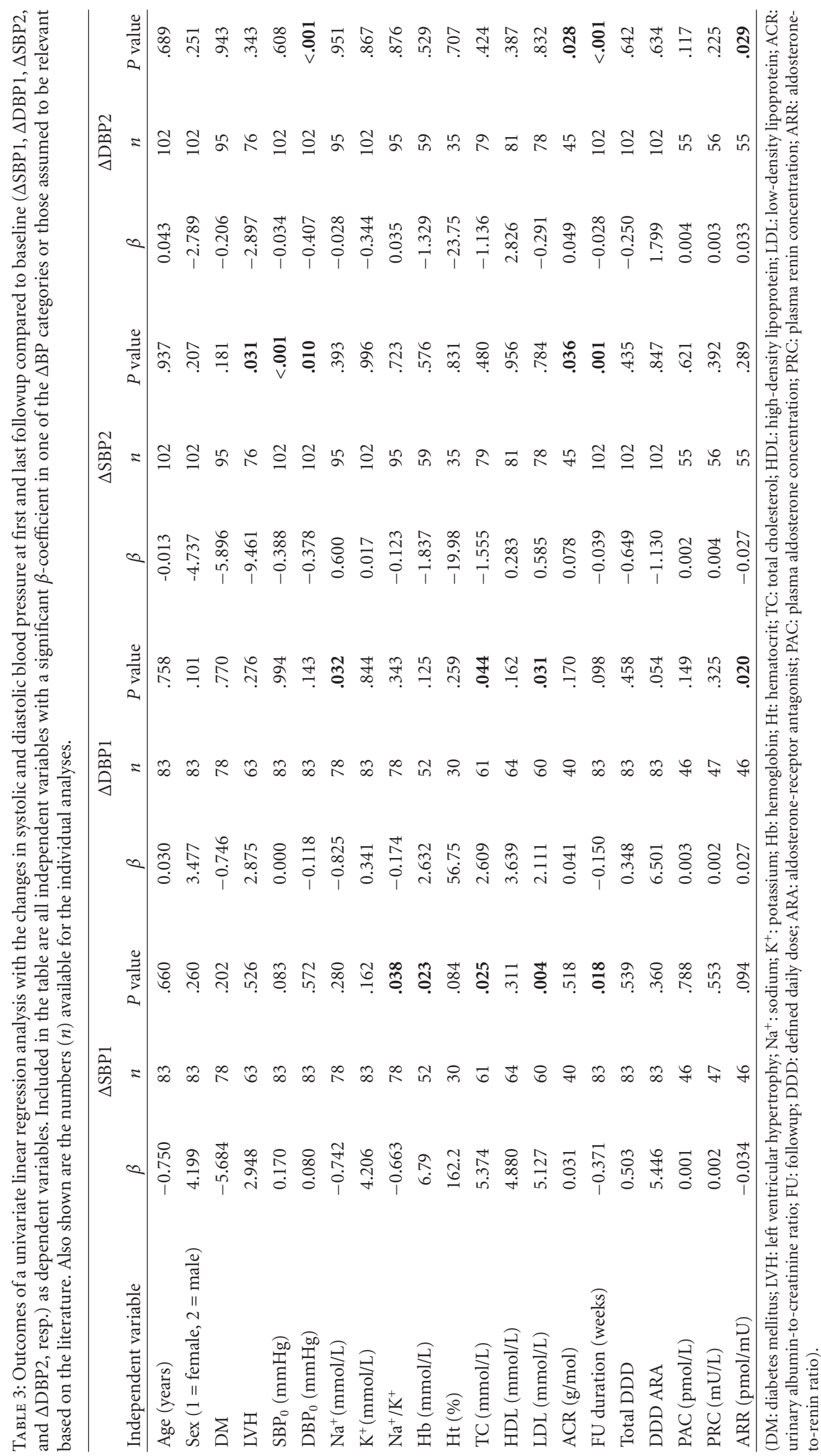




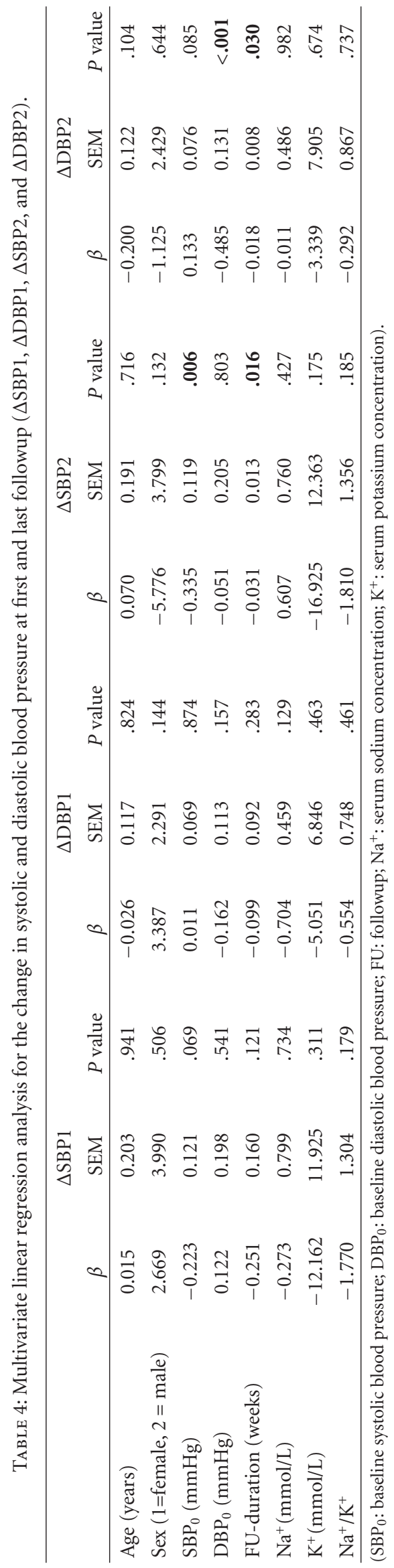


spironolactone treatment in resistant hypertension with 24hour ambulatory BP measurements, thereby eliminating a white-coat effect and at least in part also a placebo effect. In their study, SBP was reduced by $16 \mathrm{mmHg}$ and DBP by $9 \mathrm{mmHg}$.

The longest followup in all mentioned studies was 15 months. Whether the effect persists over a longer period had not yet been reported. With all the limitations of a retrospective design, our study is the first to show that the BP lowering effect of add-on ARA treatment is profound and persistent even after years of treatment.

Earlier publications have focused on identifying clinical and biochemical predictors for the BP response to ARA treatment. Several studies have shown that neither plasma renin concentration or activity, nor aldosterone or the ARR are good predictors for this response [5, 9, 17, 22, 26], although this may only hold for patients on multidrug regimens [25] related to the interfering effects of many antihypertensives on renin and aldosterone levels [28]. Low serum potassium levels have consistently been shown to be associated with a better response [19, 21, 22, 25]. Other factors potentially related to a better BP response are higher waist circumference, lower aortic pulse wave velocity [22], the absence of DM [23], and a lower baseline HDL cholesterol [21]. In a univariate linear regression analysis, we could not confirm the predictive value of the serum potassium level for BP response. However, the sodium/potassium ratio (as a potential indicator for aldosterone excess) showed a significant correlation with SBP decrease at short followup at univariate analysis. In a multivariate analysis, only higher baseline BP and longer followup duration independently predicted BP response in the long run. Potential explanations for this have been discussed earlier in this section. In our univariate analysis, also haemoglobin, total cholesterol, and LDL for short-term followup and left ventricular hypertrophy and urinary albumin-to-creatinine ratio for long-term followup were identified as potential predictors. Unfortunately, because of too many missing values, these variables were not included in the multivariate analysis to maintain enough statistical power. However, these parameters are important candidates for further studies on determinants of BP lowering by ARAs. Plasma renin and aldosterone levels were not associated with $\mathrm{BP}$ response, as has been reported earlier. In our univariate analysis, the ARR was weakly, yet significantly, associated with change in DBP. Considering the median ARR of $9.5 \mathrm{pmol} / \mathrm{mU}$ in this patient group, a beta coefficient of 0.027 is probably of little relevance. Also the number of patients with ARR available at baseline was too small to include in the multivariate analysis.

The mechanism that underlies the BP-lowering effect of add-on ARA treatment is most likely induction of natriuresis and diuresis although extrarenal effects of aldosterone blockade may also be of importance, such as a reduction in sympathetic tone and modulation of vascular tone, and in the long run a reduction in vascular stiffness may also play a role (reviewed in [29]). The clinical relevance of these extrarenal mechanisms is unknown. A cross-over trial in patients with low-renin hypertension, an elevated ARR, and a previous favourable $\mathrm{BP}$ response to spironolactone showed that even in this selected population, high-dose thiazide diuretic treatment was as effective as $100 \mathrm{mg}$ of spironolactone, strongly suggesting that natriuresis is the most important mode of action [30]. This also underscores the relevance of dietary salt reduction in resistant hypertension as has been shown elsewhere [31]. In general, ARA treatment was well tolerated and side effects were rare. In 13 patients, side effects were reported (10.6\%), most of them presenting with gynaecomastia or hyperkalemia. The occurrence of sex hormone-related side effects with spironolactone is dose dependent [32], and in many cases, these side effects can be prevented by using lower doses. When this is also not tolerated, treatment with eplerenone, being a more specific ARA with virtually no sex hormonerelated actions in therapeutic doses, can be considered.

Risk factors for hyperkalemia are advanced age, diabetes mellitus, higher baseline potassium levels [33], and advanced stage 3 nephropathy [34]. The presence of renal function impairment and concomitant use of other diuretics predisposes to the development of renal failure [33]. Frequent monitoring of serum potassium and renal function is warranted in these patients.

Our study has several limitations, the most significant one being its retrospective nature. Because of this, there is an important heterogeneity in patients, treatment, and followup. To properly assess the long-term efficacy of ARA treatment taking into account the large differences in followup, stratification to followup duration was made. This makes the analysis prone to bias with overrepresentation of patients with a good response in the group of prolonged followup. It would have been more ideal to collect patient data at several time points during the followup period, but clinical information in the written files was not always present. Furthermore, biochemical parameters, especially haemoglobin and cholesterol (including HDL and LDL) at baseline, were only available for a limited number of patients, thereby limiting their usefulness for multivariate analysis because of lack of statistical power. Also renin and aldosterone levels were only available for a subset of patients.

This study shows that long-term treatment including an ARA leads to a persistent BP reduction. Whether this is attributable to the ARA itself or to better treatment in general is an important point of consideration. As shown, BP reduction was not accompanied by an increase in total amount of antihypertensive drugs, thereby making a specific effect of the intervention with an ARA more likely. Last, the distinction between patients with EH and PA was solely based on a clinical diagnosis by the patient's physician. A formal confirmation test for PA was only performed in a proportion of the patients labelled with the diagnosis PA. The recent guidelines for the diagnosis and treatment of PA made by the Endocrine Society advise to perform a confirmation test in patients with an ARR of approximately $91 \mathrm{pmol} / \mathrm{mU}$ [35]. From the ranges in ARR reported in Table 1 , it could be deduced that some of the EH patients actually had PA and that some of the PA patients had been misdiagnosed. However, considering the substantial differences in renin, aldosterone, and potassium levels between our EH and PA patients, we think that the diagnosis was correct in most of the patients. 
With all limitations, our results are in favour of a profound and long-term BP lowering effect of ARA treatment in difficult-to-treat hypertension. To assess the magnitude of the response more accurately, a randomized, placebocontrolled trial is needed. With all evidence available, ARAs at moderate dosages are a welcome treatment option in patients with difficult-to-treat or resistant hypertension.

\section{Abbreviations}

ACR: Urinary albumin-to-creatinine ratio

ANOVA: Analysis of variance

ARA: Aldosterone-receptor antagonist

ARR: Aldosterone-to-renin ratio

BP: $\quad$ Blood pressure

DBP: Diastolic blood pressure

DDD: Defined daily dose

DM: Diabetes mellitus

ECG: Electrocardiogram

EH: Essential hypertension

HDL: High-density lipoprotein

LDL: Low-density lipoprotein

LVH: Left ventricular hypertrophy

PA: $\quad$ Primary aldosteronism

PAC: Plasma aldosterone concentration

PRC: Plasma renin concentration

RAAS: Renin-angiotensin-aldosterone system

SBP: $\quad$ Systolic blood pressure.

\section{Acknowledgment}

P. M. Jansen is financially supported by the Dutch Kidney Foundation (Grant no. C05.2151).

\section{References}

[1] R. I. Ogilvie, K. M. Piafsky, and J. Ruedy, "Antihypertensive responses to spironolactone in normal renin hypertension," Clinical Pharmacology and Therapeutics, vol. 24, no. 5, pp. 525-530, 1978.

[2] B. E. Karlberg, B. Kagedal, L. Tegler, K. Tolagen, and B. Bergman, "Controlled treatment of primary hypertension with propranolol and spironolactone. A crossover study with special reference to initial plasma renin activity," The American Journal of Cardiology, vol. 37, no. 4, pp. 642-649, 1976.

[3] J. H. Kreeft, P. Larochelle, and R. I. Ogilvie, "Comparison of chlorthalidone and spironolactone in low-renin essential hypertension," Canadian Medical Association Journal, vol. 128, no. 1, pp. 31-34, 1983.

[4] M. H. Weinberger, B. Roniker, S. L. Krause, and R. J. Weiss, "Eplerenone, a selective aldosterone blocker, in mildto-moderate hypertension," American Journal of Hypertension, vol. 15, no. 8, pp. 709-716, 2002.

[5] M. H. Weinberger, W. B. White, L. M. Ruilope et al., "Effects of eplerenone versus losartan in patients with low-renin hypertension," American Heart Journal, vol. 150, no. 3, pp. 426-433, 2005.

[6] E. D. Burgess, Y. Lacourcière, L. M. Ruilope-Urioste et al., "Long-term safety and efficacy of the selective aldosterone blocker eplerenone in patients with essential hypertension," Clinical Therapeutics, vol. 25, no. 9, pp. 2388-2404, 2003.

[7] W. B. White, A. A. Carr, S. Krause, R. Jordan, B. Roniker, and W. Oigman, "Assessment of the novel selective aldosterone blocker eplerenone using ambulatory and clinical blood pressure in patients with systemic hypertension," American Journal of Cardiology, vol. 92, no. 1, pp. 38-42, 2003.

[8] J. M. Flack, S. Oparil, J. H. Pratt et al., "Efficacy and tolerability of eplerenone and losartan in hypertensive black and white patients," Journal of the American College of Cardiology, vol. 41, no. 7, pp. 1148-1155, 2003.

[9] G. H. Williams, E. Burgess, R. E. Kolloch et al., "Efficacy of eplerenone versus enalapril as monotherapy in systemic hypertension," American Journal of Cardiology, vol. 93, no. 8, pp. 990-996, 2004.

[10] W. B. White, D. Duprez, R. S. Hillaire et al., "Effects of the selective aldosterone blocker eplerenone versus the calcium antagonist amlodipine in systolic hypertension," Hypertension, vol. 41, no. 5, pp. 1021-1026, 2003.

[11] B. Pitt, N. Reichek, R. Willenbrock et al., "Effects of eplerenone, enalapril, and eplerenone/enalapril in patients with essential hypertension and left ventricular hypertrophy: the 4E-left ventricular hypertrophy study," Circulation, vol. 108, no. 15, pp. 1831-1838, 2003.

[12] P. M. Jansen, F. Boomsma, A. H. van den Meiracker et al., "Aldosterone-to-renin ratio as a screening test for primary aldosteronism: the Dutch ARRAT study," Netherlands Journal of Medicine, vol. 66, no. 5, pp. 220-228, 2008.

[13] M. Sartori, L. A. Calò, V. Mascagna et al., "Aldosterone and refractory hypertension: a prospective cohort study," American Journal of Hypertension, vol. 19, no. 4, pp. 373-379, 2006, discussion 380 .

[14] J. Staessen, P. Lijnen, and R. Fagard, "Rise in plasma concentration of aldosterone during long-term angiotensin II suppression," Journal of Endocrinology, vol. 91, no. 3, pp. 457$465,1981$.

[15] K. J. Schjoedt, S. Andersen, P. Rossing, L. Tarnow, and H. H. Parving, "Aldosterone escape during blockade of the reninangiotensin-aldosterone system in diabetic nephropathy is associated with enhanced decline in glomerular filtration rate," Diabetologia, vol. 47, no. 11, pp. 1936-1939, 2004.

[16] A. S. Bomback and P. J. Klemmer, "The incidence and implications of aldosterone breakthrough," Nature Clinical Practice Nephrology, vol. 3, no. 9, pp. 486-492, 2007.

[17] M. K. Nishizaka, M. A. Zaman, and D. A. Calhoun, "Efficacy of low-dose spironolactone in subjects with resistant hypertension," American Journal of Hypertension, vol. 16, no. 11, part 1, pp. 925-930, 2003.

[18] J. Ouzan, C. Pérault, A. M. Lincoff, E. Carré, and M. Mertes, "The role of spironolactone in the treatment of patients with refractory hypertension," American Journal of Hypertension, vol. 15, no. 4, part 1, pp. 333-339, 2002.

[19] Y. Sharabi, E. Adler, A. Shamis, N. Nussinovitch, A. Markovitz, and E. Grossman, "Efficacy of add-on aldosterone receptor blocker in uncontrolled hypertension," American Journal of Hypertension, vol. 19, no. 7, pp. 750-755, 2006.

[20] D. A. Lane, S. Shah, and D. G. Beevers, "Low-dose spironolactone in the management of resistant hypertension: a surveillance study," Journal of Hypertension, vol. 25, no. 4, pp. 891-894, 2007.

[21] N. Chapman, J. Dobson, S. Wilson et al., "Effect of spironolactone on blood pressure in subjects with resistant hypertension," Hypertension, vol. 49, no. 4, pp. 839-845, 2007. 
[22] F. de Souza, E. Muxfeldt, R. Fiszman, and G. Salles, "Efficacy of spironolactone therapy in patients with true resistant hypertension," Hypertension, vol. 55, no. 1, pp. 147-152, 2010.

[23] E. Rodilla, J. A. Costa, F. Pérez-Lahiguera, E. Baldó, C. González, and J. M. Pascual, "Spironolactone and doxazosin treatment in patients with resistant hypertension," Revista Espanola de Cardiologia, vol. 62, no. 2, pp. 158-166, 2009.

[24] C. Saha, G. J. Eckert, W. T. Ambrosius et al., "Improvement in blood pressure with inhibition of the epithelial sodium channel in blacks with hypertension," Hypertension, vol. 46, no. 3, pp. 481-487, 2005.

[25] A. Mahmud, M. Mahgoub, M. Hall, and J. Feely, "Does aldosterone-to-renin ratio predict the antihypertensive effect of the aldosterone antagonist spironolactone?" American Journal of Hypertension, vol. 18, no. 12, pp. 1631-1635, 2005.

[26] L. M. Prisant, H. Krum, B. Roniker, S. L. Krause, K. Fakouhi, and W. He, "Can renin status predict the antihypertensive efficacy of eplerenone add-on therapy?" Journal of Clinical Pharmacology, vol. 43, no. 11, pp. 1203-1210, 2003.

[27] WHO Collaborating Centre for Drug Statistics Methodology, "ATC/DDD Index 2010," http://www.whocc.no/atc_ddd_ index/.

[28] P. Mulatero, F. Rabbia, A. Milan et al., "Drug effects on aldosterone/plasma renin activity ratio in primary aldosteronism," Hypertension, vol. 40, no. 6, pp. 897-902, 2002.

[29] P. M. Jansen, A. H. J. Danser, B. P. Imholz, and A. H. van den Meiracker, "Aldosterone-receptor antagonism in hypertension," Journal of Hypertension, vol. 27, no. 4, pp. 680691, 2009.

[30] S. J. Hood, K. P. Taylor, M. J. Ashby, and M. J. Brown, "The spironolactone, amiloride, losartan, and thiazide (SALT) double-blind crossover trial in patients with low-renin hypertension and elevated aldosterone-renin ratio," Circulation, vol. 116, no. 3, pp. 268-275, 2007.

[31] E. Pimenta, K. K. Gaddam, S. Oparil et al., "Effects of dietary sodium reduction on blood pressure in subjects with resistant hypertension: results from a randomized trial," Hypertension, vol. 54, no. 3, pp. 475-481, 2009.

[32] X. Jeunemaitre, G. Chatellier, C. Kreft-Jais et al., "Efficacy and tolerance of spironolactone in essential hypertension," American Journal of Cardiology, vol. 60, no. 10, pp. 820-825, 1987.

[33] K. P. Tamirisa, K. D. Aaronson, and T. M. Koelling, "Spironolactone-induced renal insufficiency and hyperkalemia in patients with heart failure," American Heart Journal, vol. 148, no. 6, pp. 971-978, 2004.

[34] N. Khosla, R. Kalaitzidis, and G. L. Bakris, "Predictors of hyperkalemia risk following hypertension control with aldosterone blockade," American Journal of Nephrology, vol. 30, no. 5, pp. 418-424, 2009.

[35] J. W. Funder, R. M. Carey, C. Fardella et al., "Case detection, diagnosis, and treatment of patients with primary aldosteronism: an endocrine society clinical practice guideline," Journal of Clinical Endocrinology and Metabolism, vol. 93, no. 9, pp. 3266-3281, 2008. 


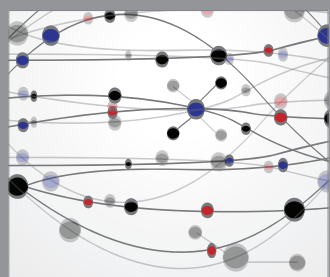

The Scientific World Journal
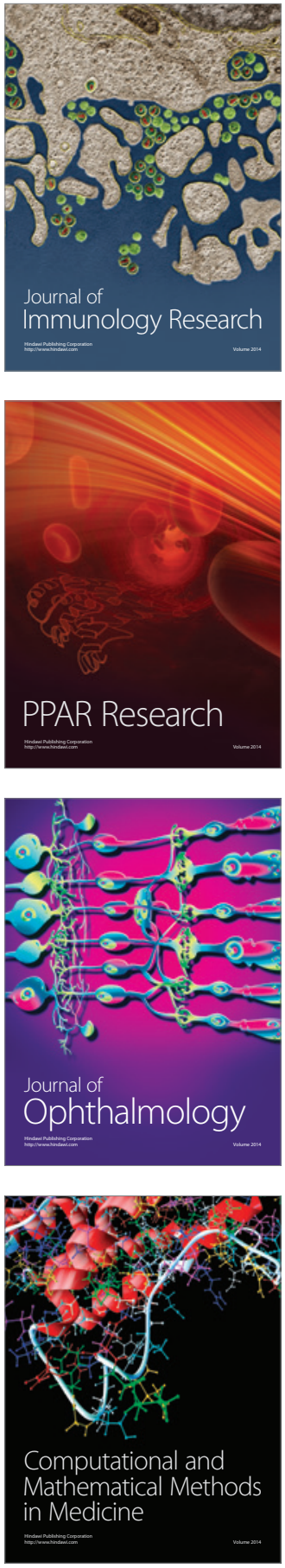

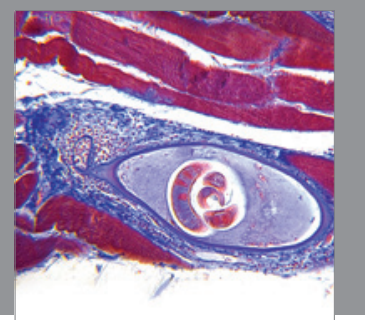

Gastroenterology

Research and Practice
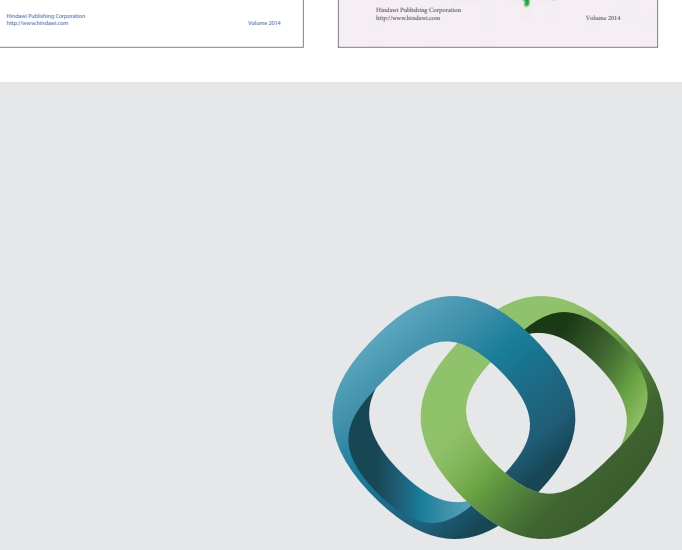

\section{Hindawi}

Submit your manuscripts at

http://www.hindawi.com
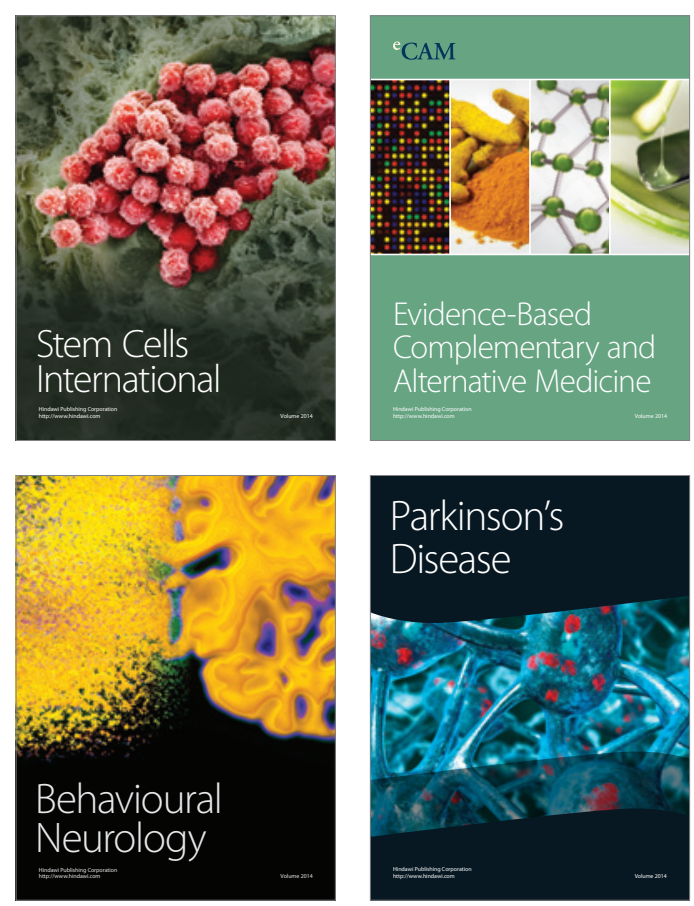

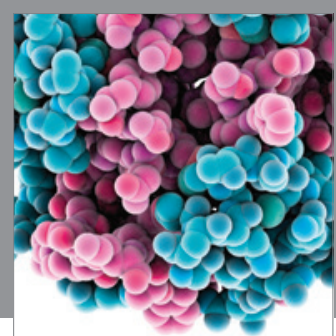

Journal of
Diabetes Research

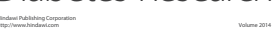

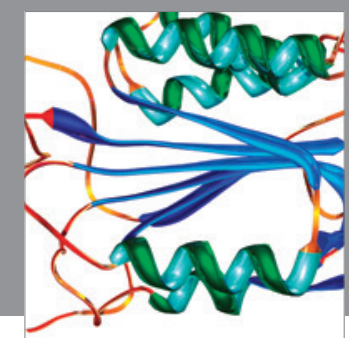

Disease Markers
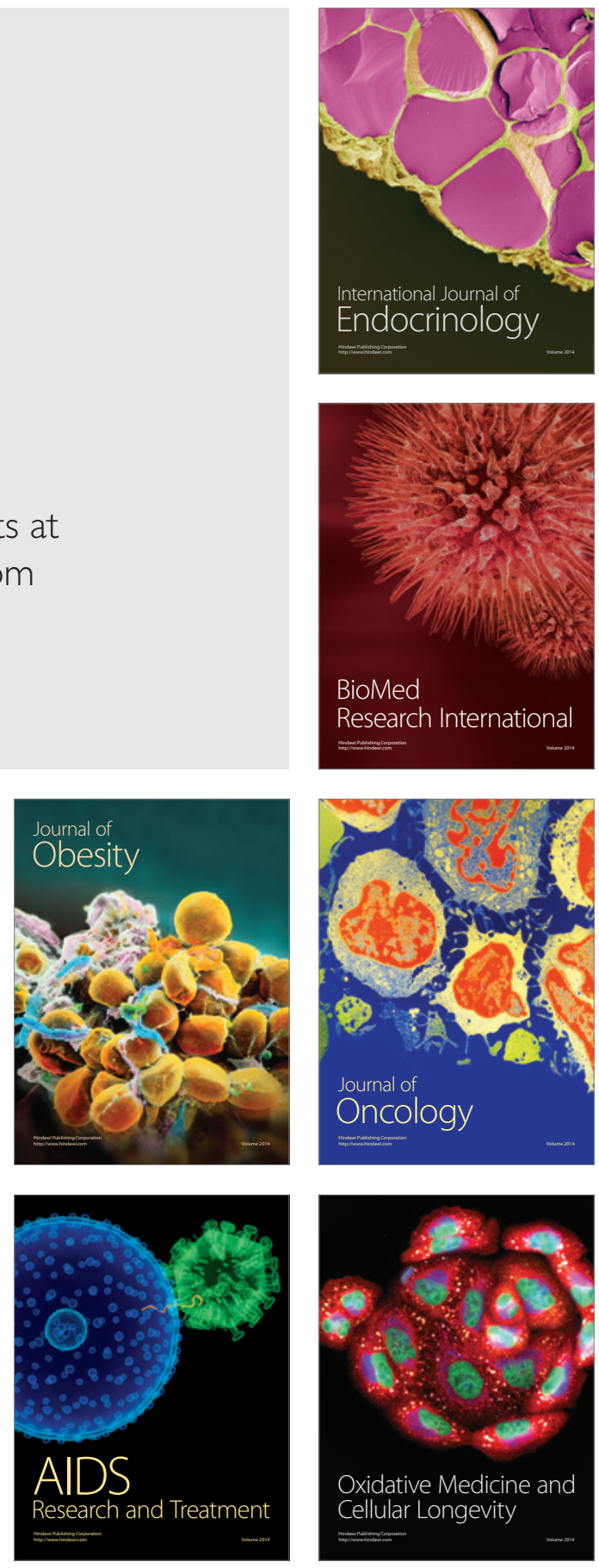